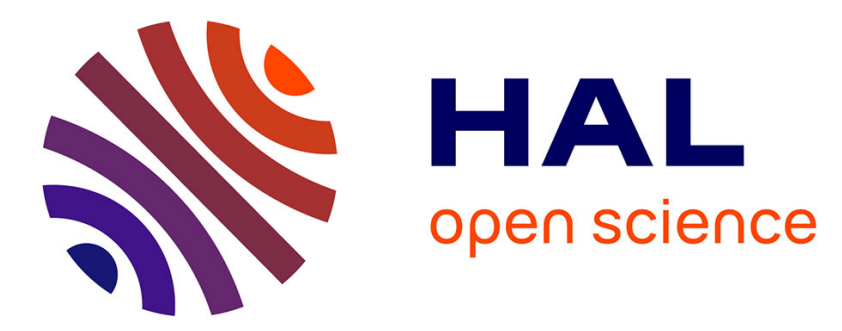

\title{
Sur les aménagements forestiers en Afrique de l'Ouest soudano-sahélienne
}

Gérard Soulères

\section{To cite this version:}

Gérard Soulères. Sur les aménagements forestiers en Afrique de l'Ouest soudano-sahélienne. Revue forestière française, 1991, 43 (2), pp.163-174. 10.4267/2042/26191 . hal-03425187

\section{HAL Id: hal-03425187 \\ https://hal.science/hal-03425187}

Submitted on 10 Nov 2021

HAL is a multi-disciplinary open access archive for the deposit and dissemination of scientific research documents, whether they are published or not. The documents may come from teaching and research institutions in France or abroad, or from public or private research centers.
L'archive ouverte pluridisciplinaire HAL, est destinée au dépôt et à la diffusion de documents scientifiques de niveau recherche, publiés ou non, émanant des établissements d'enseignement et de recherche français ou étrangers, des laboratoires publics ou privés. 


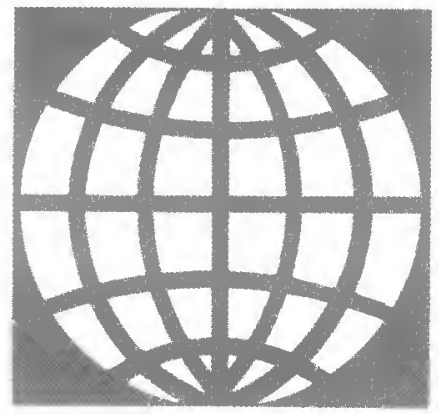

\section{chronique internationale}

\section{SUR LES AMÉNAGEMENTS FORESTIERS EN AFRIQUE DE L'OUEST SOUDANO-SAHÉLIENNE}

G. SOULÈRES

En forêt aussi, il y a des modes. Ainsi dans les années soixante et soixante-dix, développement forestier et satisfaction des besoins en bois se traduisaient par reboisement dans la zone sahélienne de l'Afrique occidentale.

De nombreuses plantations furent programmées et réalisées, en Eucalyptus camaldulensis par exemple. Elles devaient donner, à l'issue d'une courte rotation, 6 à 10 ans, des volumes importants des produits qui commençaient à manquer aux populations (bois de feu, bois de service), correspondant souvent à une production espérée égale ou supérieure à 10 mètres cubes par hectare et par an.

Hélas, ces espoirs ne furent que très rarement exaucés. Beaucoup de plantations, faute d'entretien, démarrèrent mal, puis stagnèrent. D'autres, mieux entretenues et même dans certains cas irriguées, ne dépassèrent quand même pas un ou deux mètres cubes par hectare et par an.

Des regarnis furent réalisés dans de nombreux cas en espèces moins exigeantes: Prosopis sp, Acacia sp. Azadirachta indica. Les boisements furent de plus en plus prévus en espèces frugales et en essences locales adaptées. Et aussí, les aménagements de forêts nafurelles se multiplièrent, remplaçant les projets de reboisement dévalorisés. Ils correspondent maintenant à une bonne part des efforts réalisés en matière de développement forestier. Bien sûr, il y en avait eu auparavant, le plus ancien ètant peut-être celui de la forêt de Bandia, au Sénégal, rédigé en 1954. 


\section{G. SOULÉRES}

II est maintenant possible et intéressant de faire un premier point sur les aménagements élaborés et appliqués, et malheureusement, sur les mauvais résultats obtenus pour la plupart d'entre eux, en cherchant à en préciser les raisons.

Mais avant de faire ce point, il paraît souhaitable de rappeler rapidement les principaux caractères des forêts objet de cet article.

Elles sont dispersées sur une bande de près de 3000 kilomètres de longueur et d'environ 350 kilomètres de largeur moyenne entre le Sénégal à l'ouest et le Niger à l'est, via le Mali, le Burkina Faso, qui affleure le nord de la Guinée et de la Côte-d'Ivoire et mord sur le Togo, le Bénin et le Nigéria. En additionnant les chiffres des différents États, on obtient une surface totale supérieure à 25 millions d'hectares. En fait, elle est vraisemblablement nettement inférieure. Beaucoup de forêts ont déjà disparu.

On voit que les forêts, qui nous intéressent ici, ne sont pas toutes celles qui occupent la zone sahélienne. Ce sont celles d'entre elles qui y présentent les potentialités les plus fortes, que l'on qualifie de soudano-sahéliennes.

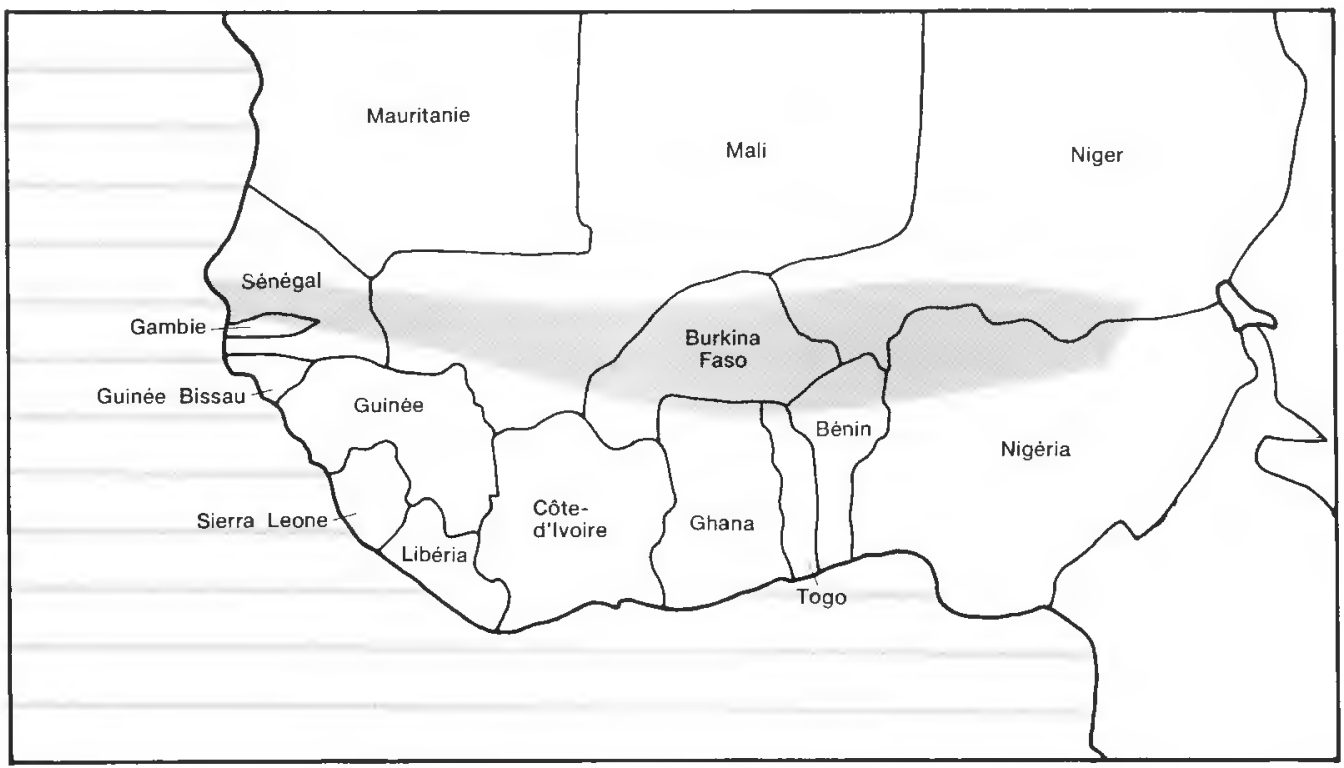

Leur aire est limitée en gros au nord par l'isohyète 600 millimètres, devenue avec la sécheresse des deux dernières décennies (1968-1989) l'isohyète 500 millimètres. Au-delà, vers le nord, s'étendent des formations purement arbustives, où dominent les épineux, sur la steppe.

Vers le sud, elles sont limitées par le climat soudanien, c'est-à-dire en gros par l'isohyète 800850 devenue 700 , qui correspond à des forêts plus denses, hors de la zone sahélienne.

L'importante baisse des pluies, qui peut varier de 10 à $30 \%$ suivant les stations météorologiques, n'affecte pas également toutes les années. Les années sèches sont souvent regroupées, par exemple 1968-1973, séparées par des périodes de rémission.

Cette sécheresse a eu des effets sur la végétation. Certaines essences ont disparu ou se sont raréfiées. Le meilleur exemple, pour toute la région, est le Kapokier. 
Mais ce qui caractérise le plus le climat, au-delà des précipitations, c'est l'importance du déficit de l'évaporation dont souffre particulièrement la végétation, qui a dû s'adapter. On sait qu'il s'agit là de la différence entre l'évaporation correspondant à une production optimum et celle permise par la ressource en eau. Il est dû à la fois à la longueur de la saison sèche (7 à 8 mois), aux fortes températures, à l'état hygrométrique très bas, aux vents desséchants (harmattan).

Les sols apparaissent, pour l'essentjel, homogènes dans toute notre zone, caractérisés par un faible niveau de fertilité chimique, une texture le plus souvent sableuse ou sablo-limoneuse ou limoneuse, un taux de matière organique faible et une mauvaise structure. Iis sont aussi souvent peu profonds, sur cuirasse latéritique. Les deux éléments qui font varier les aptitudes sont la profondeur et la teneur en argile.

Les meilleurs sols occupent les lits des marigots (cours d'eau le plus souvent temporaires) et les dépressions. Ils sont à la fois enrichis en éléments fins et frais plus longtemps.

De tels sols sont très sensibles aux différentes formes de l'érosion, d'autant plus que la couverture végétale qui les protégeait s'éclaircit. Ils souffrent de l'érosion éolienne, de l'érosion par ruissellement (très longs versants à pente faible). Les éléments sont dissociés par la chute des gouttes d'eau (effet "splash»). À la partie supérieure se constitue une croûte durcie qui rend plus difficile ou même impossible le développement des semis.

Les forêts, forêts claires rarement, savanes boisées ou arborées plus souvent, savane arbustive et steppe boisée parfois, sous l'effet de la dégradation, s'organisent en deux étages:

- Un étage dominant, que l'on appelle souvent futaie, d'essences plus ou moins variées, plus ou moins dense.

Les arbres peuvent atteindre une quinzaine de mètres ou plus (Khaya senegalensis, Prosopis africana, Anogeissus leiocarpus, Pterocarpus erinaceus) dans des formations en général complexes ou mélangées, ou culminer difficilement vers 7 ou 8 mètres, ou même moins (4 ou 5 mètres). II s'agit le plus souvent dans ces derniers cas de formations presque pures ou tout au moins où certaines essences dominent très largement (Isoberlinia doka, qui peut être plus haut, Sclerocarya birrea).

Les formations de marigot où la futaie est exclusive, de structure irrégulière, occupent une place particulière par leur densité, leur variété, leur dynamisme.

- Un sous-étage d'espèces possédant en général une bonne aptitude à rejeter, ce qui lui vaut le nom de taillis (Combretum spp., petits Acacias,...).

Les volumes totaux sur pied pour des forêts en équilibre varient suivant les milieux entre 30 et 80 stères bois fort par hectare, plus pour certaines forêts galeries.

La production, pour des forêts en relativement bon état, est comprise entre 0,6 et 1,5 ou 2 stères par hectare ou par an. C'est essentiellement du bois de feu et de service (piquets, petits poteaux, manches d'outils). Les besoins sont énormes et mal satisfaits.

Mais, en fait, on constate sur le terrain que beaucoup de ces "forêts " ne sont plus que des formations résiduelles. Le peuplement subsistant dominant est composé d'essences qui ont été conservées lors des défrichements, ou même favorisées, parce qu'elles pouvaient donner des fruits ou des produits utiles (Cordyla pinnata, Sterculia setigera, Sclerocarya birrea). Les autres essences disparaissent progressivement.

Dans l'étage dominé, les essences subsistantes ont été sélectionnées par leur aptitude à rejeter (Combretum spp.). Et certaines autres espèces apparaissent puis se développent au fur et à mesure des progrès de la dégradation, finissant par constituer une part importante du recouvrement. C'est le cas de Guiera senegalensis, que dans certaines forêts on est amené à considérer maintenant comme une essence du taillis. 


\section{G. SOULERES}

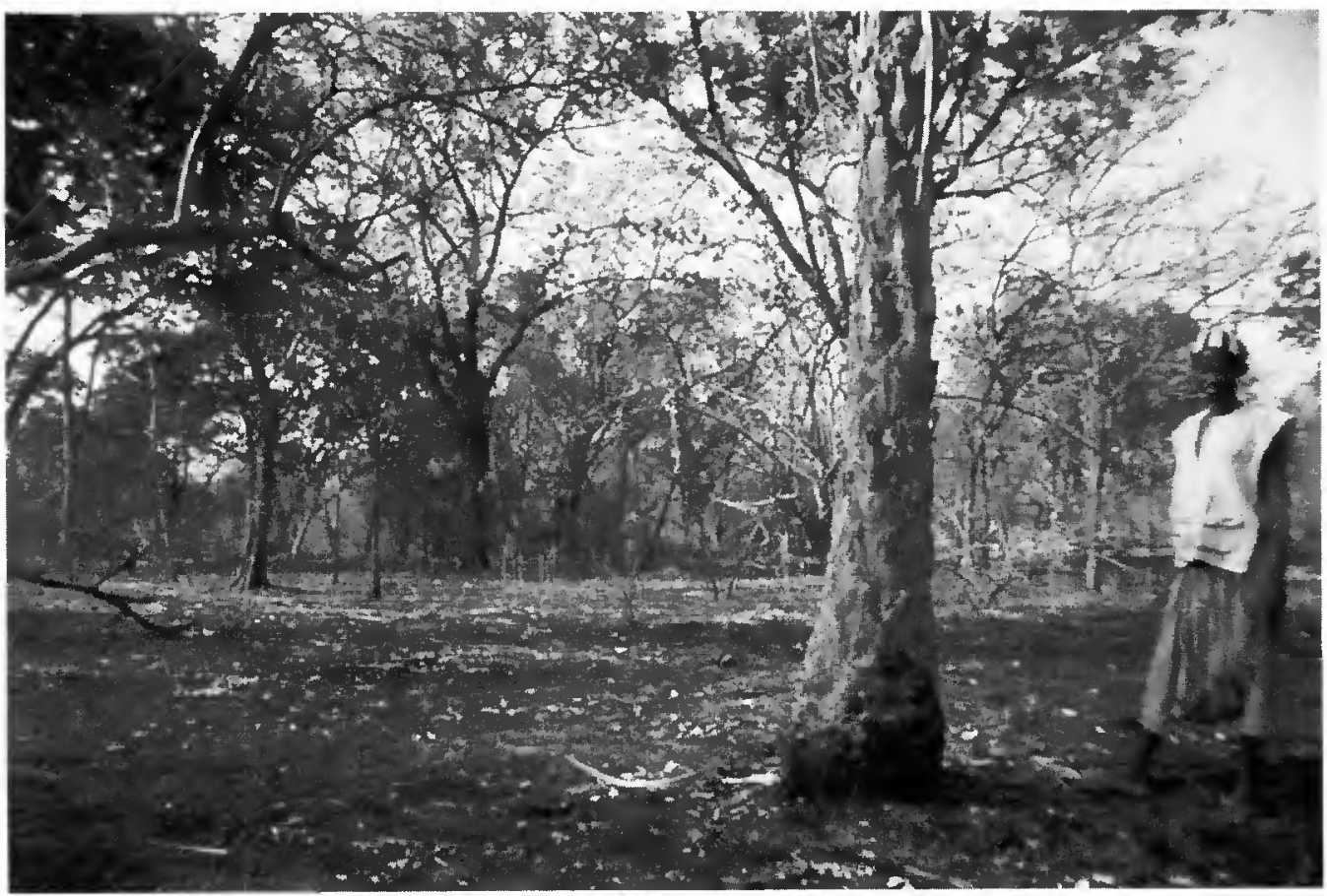

Sénégal. Forêt de Dabo (Haute-Casamance). Après le passage du teu.

Niger. Forêt de Dan Kada Dodo. Un stade avancé de la dégradation.

Photos G. Soulères

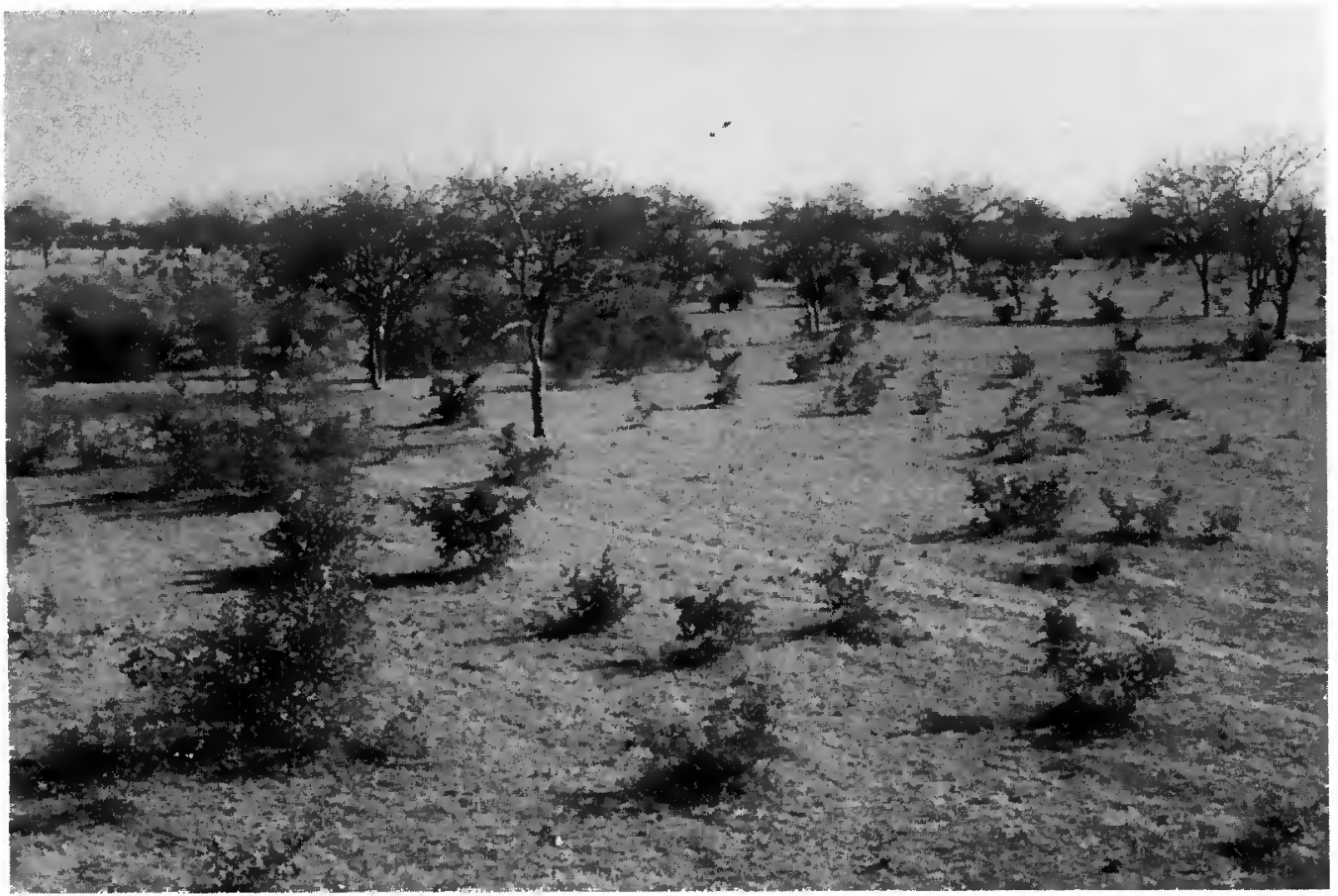


Le processus de dégradation (défrichement, surexploitation, feu, surpâturage...) est commandé par l'augmentation de la densité de la population et celle corrélative des troupeaux. Vers 15 habitants au $\mathrm{km}^{2}$, la forêt est encore en équilibre ou en bon état. Vers 50-60 habitants au $\mathrm{km}^{2}$, elle est proche de sa disparition. Avec un taux d'augmentation de la population rurale généralement proche de $3 \%$, il faut ainsi à peu près 40/50 ans pour que le processus s'accomplisse. Mais il peut être beaucoup plus rapide en cas de mauvais traitement prolongé ou dans les secteurs les moins favorisés. Il a aussi été accéléré par la sécheresse qui a réduit la capacité de réaction de la forêt.

\section{$\stackrel{* * *}{*}$}

Comme il a été dit, dans ce milieu difficile, de nombreuses forêts ont maintenant été aménagées, de nombreux aménagements ont été appliqués au cours de durées suffisamment longues pour que l'on puisse en apprécier les résultats. Ceux-ci sont fréquemment médiocres. L'application d'un aménagement, dans le contexte existant, a souvent entraîné en fait, une régression de l'état forestier. Il est important de chercher à comprendre les raisons de ces échecs. Certaines explications paraissent pouvoir être avancées. Parmi elles, quatre ont été retenues pour cet article qui sont présentées dans les quatre chapitres suivants. Elles sont de natures varièes, liées aussi bien à des facteurs que l'on peut qualifier de techniques, qu'à d'autres en relation avec les populations et la situation particulière de beaucoup de forêts.

II n'est pas question de proposer des solutions, elles ne peuvent être trouvées qu'au coup par coup. L'auteur est aussi bien conscient des conséquences de la faiblesse des Services.

\section{LES AMÉNAGEMENTS N'ONT PAS ÉTÉ SUFFISAMMENT ADAPTÉS À L'ÉTAT ET AU DYNAMISME DES FORÉTS}

Ils ont été conçus pour l'exploitation. Très souvent d'ailleurs, on a commencé par estimer les besoins en bois d'un centre avant de chercher à les satisfaire par l'aménagement des forêts les plus proches.

Les prélèvements ainsi organisés sont venus s'ajouter aux prélèvements de toute nature que subissait déjà la forêt : défrichement, feux, exploitations en délit, mort naturelle,... Les forêts de la zone sont toutes en état de dégradation, plus ou moins avancé. Et parallèlement à la dégradation du peuplement, on observe une dégradation de l'ambiance forestière qui devient de moins en moins favorable.

Dans une forêt en équilibre, la régénération est un phénomène continu. Chaque année, de nouveaux semis apparaissent et survivent. $\dot{A}$ un stade plus avancé de la dégradation, la régénération devient un phénomène discontinu, liẻe à des années plus favorables. Puis elle n'est plus possible.

Suivant la dégradation des peuplements, suivant la dégradation de l'ambiance forestière, on peut ainsi de moins en moins demander à la forêt pour assurer sa survie. Bientôt, on ne peut plus beaucoup compter sur elle. Ses souches ne peuvent que rejeter et encore dans un milieu devenu défavorable, même aux rejets.

L'intervention humaine est nécessaire pour assurer la pérennité de la forêt. Et c'est elle qui achète en quelque sorte le droit de couper.

Sur le chemin, représenté plus loin, qui conduit ainsi de la forêt en équilibre, au sol non encroûté, aux divers stades de la dégradation, devraient correspondre des traitements différents, exigeant des interventions de plus en plus lourdes: 
par exemple:

exploitation / régénération naturelle

exploitation / enrichissement

enrichissement / exploitation

enrichissement sans exploitation

reconstitution

LES DIVERS TRAITEMENTS

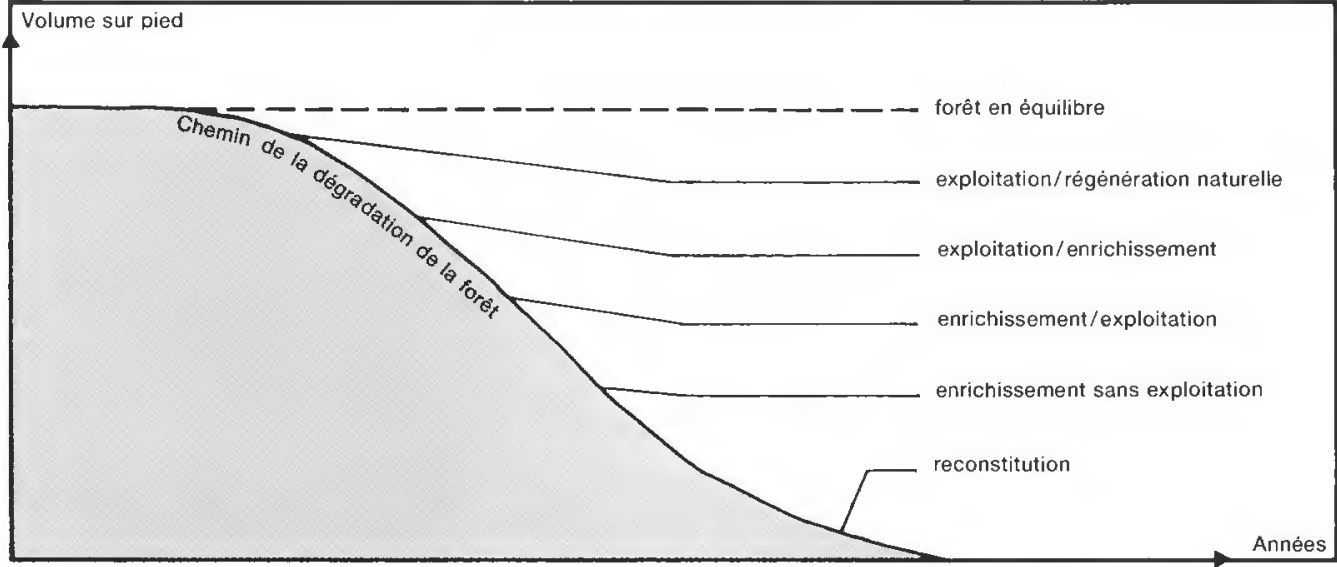

À partir d'un certain stade de dégradation, correspondant à une forêt déjà mitée, on ne peut plus commencer par couper. Réduire le peuplement sur pied ne pourrait qu'accélérer sa dégradation et celle du milieu et par là hâter la disparition de la forêt.

Dans le traitement enrichissement/exploitation, on commence donc par enrichir, en prenant en quelque sorte appui sur les éléments subsistants du peuplement initial, qui rendent le milieu moins défavorable (brise-vent).

Ce n'est qu'après que les arbres installés en enrichissement auront atteint un développement suffisant que l'on pourra exploiter certains des éléments du peuplement initial, dont les rejets seront protégés par les nouveaux arbres et arbustes.

Dans un stade encore plus dégradé, on ne peut qu'enrichir sans prévoir une exploitation dans l'aménagement élaboré. Ce n'est que dans l'aménagement suivant, éventuellement, que celle-ci pourra être programmée après un vieillissement suffisant, si les conditions maintenues jusque-là permettent le progrès.

La reconstitution enfin correspond à un stade de dégradation ultime où un peuplement doit pratiquement être recréé dans son entier par des techniques adaptées.

Un progrès sensible a été accompli dans les derniers projets. La coupe rase, qui laissait un milieu hostile, aussi bien pour une régénération naturelle hypothétique que pour le développement des rejets, n'est plus préconisée. Le modèle de coupe le plus souvent proposé est basé sur la distinction des deux étages de végétation :

- l'étage de futaie qui est surtout parcouru par une coupe sanitaire ;

- l'étage dominé qui doit être recépé et rejeté pour reconstituer le volume initial.

Au total, la moitié ou un peu plus du volume total, dont toutes les essences présentant un intérêt particulier pour les populations, reste en place. Et les coupes sont fréquemment suivies de plantations. 


\section{LA CAPACITÉ DES PEUPLEMENTS À REVENIR APRÈS EXPLOITATION À UNE SITUATION PROCHE DE L'ÉTAT INITIAL A ÉTÉ EN GÉNÉRAL SURESTIMÉE (1)}

C'est cette capacité qui permet un second cycle de coupe et assure la pérennité de l'exploitation de la forêt. Cette reconstitution du volume de départ était en général surtout attendue du développement des rejets et, aussi, d'une régénération naturelle qui était affirmée sans que sa possibilité ait été réellement vérifiée ou sans que les conditions nécessaires pour l'assurer aient été mises en place.

II est certain que le développement des rejets est très rapide dans la période qui suit le recépage. Ils parviennent très vite à une hauteur de 2 ou 3 mètres. Les essais mis en place dans plusieurs États et surtout suivis les toutes premières années le montrent bien.

Mais la croissance ne tarde pas à se ralentir et ses résultats à plafonner à un âge variable suivant les essences. Peut-être $6 / 8$ ans pour les Combretum spp., peut-être $8 / 10$ ans pour Acacia seyal?

Le recépage a aussi eu pour effet d'augmenter sensiblement le nombre de tiges par souche. Le volume moyen de chaque tige est donc nettement plus faible que dans le peuplement initial et beaucoup, lors d'une deuxième exploitation, ne seront même pas prélevées car leur volume unitaire est trop faible.

Cette évolution est bien mise en évidence par les statistiques d'exploitation dans le cas de la première forêt aménagée, celle de Bandia (Sénégal), dont certaines parcelles ont connu trois révolutions.

Les volumes moyens à l'hectare récoltés au cours de chaque révolution sont donnés ci-après :

Forêt classée de Bandia. Volumes moyens par hectare récoltés à chaque révolution pour la série $\alpha$ bois de feu *

Exploitations antérieures à l'aménagement $(1938-1953) \ldots \ldots \ldots \ldots \ldots$ st $/$ ha, en coupe rase

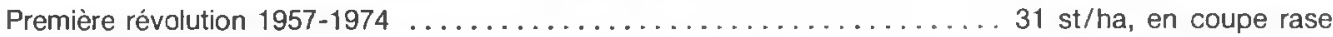

Deuxième révolution (partielle) à partir de 1975 et jusqu'en $1980 \ldots \ldots \ldots$ moins de $15 \mathrm{st} / \mathrm{ha}$,

en coupe rase

(Données fournies par l'Administration forestière sénégalaise, 1985).

Et la troisième révolution n'a d'ailleurs pas été conduite à son terme. L'aménagement a été remplacé par un projet de reboisement qui a connu les mêmes vicissitudes que la plupart d'entre eux.

Et pourtant, la forêt de Bandia n'est pas particulièrement défavorisée. II y tombait 650 à 700 millimètres qui sont devenus un peu moins de 600 millimètres au cours des deux dernières décennies, du fait de la sécheresse. Et les sols ne sont pas plus mauvais qu'ailleurs (plaine de la Somone et de son affluent).

En fait, si l'on veut reconstituer le volume, il est nécessaire de faire appel à la régénération sexuée, naturelle ou artificielle.

Naturelle, elle n'est possible que si la forêt n'est pas trop dégradée, au tout début du processus qui conduit à sa disparition. Les semenciers doivent être respectés et un milieu plus favorable à l'apparition et au développement des semis doit être créé : rassemblement des rémanents autour des semenciers ou suivant des courbes de niveaux, façons culturales dans les vides, en particulier pour casser la croûte qui s'est souvent formée.

(1) Cette déclaration péremptoire n'est pas une critique adressee aux forestiers qui ont agi. C'est parce qu'ils ont agi qu'il est maintenant possible d'apprécier. 


\section{G. SOULĖRES}

Plus souvent, il est nécessaire de faire appel à la régénération artificielle, par exemple par semis d'essences adaptées, après un traitement préalable des graines qui assure une germination rapide. Mais les "trous de sécheresse "font qu'il est parfois nécessaire de semer plusieurs fois dans la même parcelle au cours de la saison des pluies.

Les plantations d'espèces locales, surtout celles dont certains sous-produits sont appréciés, ou d'essences exotiques, soigneusement testées, sont souvent aussi nécessaires. II est maintenant admis qu'il vaut mieux éviter les plantations pures et planter plusieurs essences en mélange pied à pied. Les densités généralement retenues (250 à 600 plants/ha) le permettent.

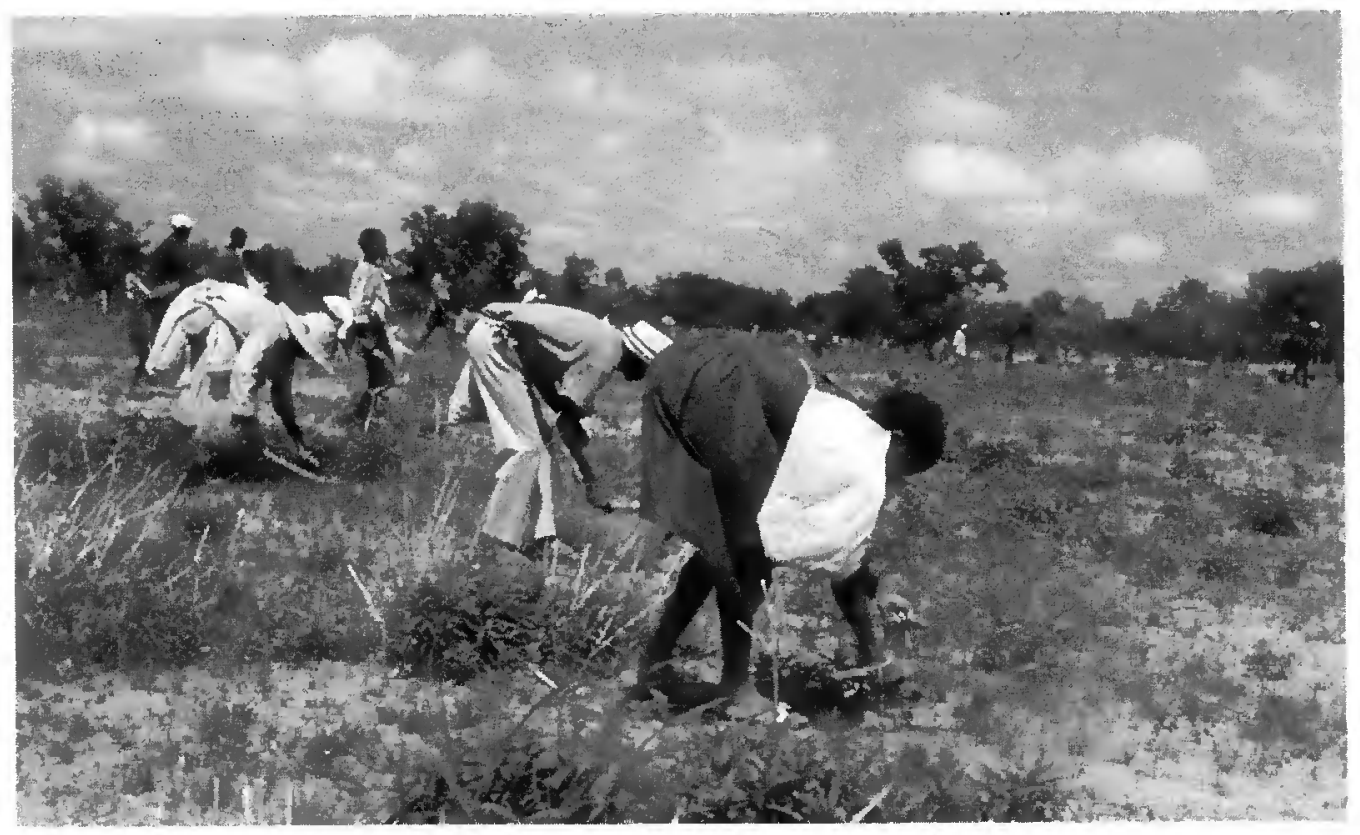

Photo G. Soulères

Semis dans une clairière autrefois cultivée. Burkina Faso. Forêt de Nazinon (Sissili).

Mais les jeunes plants et les rejets ont à craindre des ennemis qui peuvent les faire disparaître ou, au moins, retarder fortement leur croissance :

- Le parcours. Les petits ligneux ne sont pour la plupart pas consommés au cours de la saison des pluies mais en fin de saison sèche, ce qui rend leur rétablissement plus difficile. II est très facile de proclamer la mise en défens d'une parcelle. Il est beaucoup plus difficile de la faire respecter.

- Le feu. Beaucoup de forêts soudano-sahéliennes sont parcourues chaque année sur la quasi-totalité de leur surface. Les incendies sont d'autant plus dommageables qu'ils sont tardifs. Des moyens sont mis en œuvre pour les empêcher ou réduire leurs effets: les feux précoces, les pare-feu, l'organisation des populations.

Mais la plupart des forestiers considèrent avec réticence les pare-feu entretenus, préférant des pare-feu verts, constitués par exemple de fruitiers (Anacardier, dans le Sud de la zone). Ils doivent être eux-mêmes défendus contre le feu les premières années mais deviennent vite capables de résister par leur densité et le vide qu'ils font sous eux.

De toutes façons, il ne sert à rien de semer ou de planter si les jeunes plants courent des risques sérieux d'être détruits par le feu. 


\section{LES POPULATIONS VOISINES DE LA FORẼT N'ONT PAS EN GÉNÉRAL ÉTÉ ASSOCIÉES D'UNE MANIĖRE SATISFAISANTE À LA MISE EN VALEUR DE LA FORÊT, QUE REPRÉSENTE L'APPLICATION DE L'AMÉNAGEMENT}

Dans la plupart des pays, le propriétaire de la plus grande partie des forêts, au moins, est l'État. Le classement a été mal ressenti par les populations voisines. II ne leur restait plus que quelques droits d'usage limités. Le souvenir de la situation antérieure, beaucoup plus satisfaisante, subsiste trente ou quarante ans après, d'autant plus que les besoins des populations ont bien augmenté avec leur densité.

Dans ces conditions, le souci affirmé d'associer les populations à l'application de l'aménagement est malheureusement souvent resté une déclaration d'intention.

La situation la plus mal ressentie a été la concession de la coupe du bois à des exploitants ou à des charbonniers étrangers. Les usagers paraissent ainsi perdre tout droit à récupérer leur forêt. Faire intervenir les habitants voisins en tant que salariés, en argent ou en vivres, du Programme alimentaire mondial, a presque toujours donné des résultats médiocres. Le salaire peut aussi confirmer l'expropriation. Dans de nombreux cas, les réactions des usagers ont été violentes, en particulier par le feu.

La solution maintenant mise en œuvre presque partout est l'association des usagers regroupés en coopératives et tirant de leur travail un fruit proportionnel à leurs efforts.

La règle du partage des recettes doit être clairement énoncée: tant pour le coopérateur, tant pour l'État (permis de coupe), tant pour un fonds d'amélioration de la forêt et tant, parfois, pour un fonds de solidarité géré par les coopérateurs, pour eux. Et surtout cette règle doit être rapidement mise en œuvre. Il ne doit pas s'écouler trop de temps entre le travail et la perception de l'argent. L'usager satisfait, qui sait que sa forêt peut être pour lui source de bénéfices substantiels, comprendra l'intérêt de sa pérennité. II pourra beaucoup plus facilement être associé aux actions entreprises pour l'assurer.

Un autre problème est celui de la commercialisation. Des projets ont voulu aller jusqu'au bout, jusqu'à la vente au détail en ville. Ils se sont heurtés aux commerçants en bois, puissants et qui paraissent presque partout un élément incontournable de la filière. La régularité de l'approvisionnement, bien mieux assurée que par leurs circuits habituels, peut les interesser même si le prix doit être un peu plus élevé, surtout lors de l'hivernage, quand de nombreux chemins deviennent impraticables.

Mais aussi, il est souvent apparu difficile de faire coexister les deux filières pour l'approvisionnement d'un même centre :

- celle, antérieure, dans laquelle le commerçant achẻte au prix qu'il veut au bûcheron, avec ou sans permis. Il contrôle le transport et la vente en ville qui sont les chaînons essentiels ;

- celle, nouvellement mise en place, où le commerçant achète à la coopérative, à un prix plus élevé, car le coût de production est plus fort.

Des difficultés proviennent aussi du fait que le bois ètant un produit de première nécessité (90 à $95 \%$ de la consommation d'énergie de l'habitant moyen), beaucoup de gouvernements le taxent à un prix très bas qui rend encore plus difficile la rémunération des coopérateurs.

Mais si le bois apparaît à l'aménagiste comme le premier produit susceptible d'associer l'usager, il est loin d'être le seul. Quand il manque, des aménagements peuvent être basés, en totalité ou en partie, sur d'autres productions de la forêt: la culture, le fourrage, l'apiculture, les produits de cueillette, ...

À terme, d'ailleurs, l'association des populations ne peut se faire pleinement que s'il y a intégration de l'aménagement forestier à l'ensemble des activités de la population. En effet, il 


\section{G. SOULĖRES}

paraît souvent difficile de laisser insatisfaits certains des besoins importants des usagers, mis en évidence, mais que l'application de l'aménagement ne peut combler. Ce peuvent être le besoin en terre, le besoin en bois de chauffage ou de service. quand la forêt ne peut plus en produire, le besoin fourrager, ... Ces besoins peuvent faire l'objet de projets d'accompagnement de l'aménagement, installés hors forêt, faisant le plus souvent appel à l'intensification et qui permettent de débloquer la situation. Bien sûr, beaucoup de projets d'accompagnement doivent associer les forestiers aux représentants d'autres services.

Dans plusieurs États enfin, les échecs en matière d'association des populations amènent à poser la question: ne faut-il pas transférer la propriété des forêts de l'État à des collectivités locales qui seraient mieux à même d'intéresser les usagers ? Est-ce bien sûr ?

Et aussi, l'État ne doit-il pas conserver la responsabilité fondamentale de la pérennité de la ressource forestière? II paraît difficile qu'il puisse s'en décharger sur des organismes dont les moyens, dans tous les domaines, sont inférieurs aux siens. Et, s'il y avait soumission à un statut foncier adapté, la co-gestion étant assurée par un service national, quelle serait la différence ?

\section{LES AMÉNAGEMENTS N'ONT PAS SUFFISAMMENT PRIS EN COMPTE ET ORGANISÉ L'UTILISATION DES PARCOURS FORESTIERS}

Et pourtant ceux-ci, pour la quasi-totalité des forêts de la zone, sont à même de procurer des recettes globales supérieures ou très supérieures à celles du bois, au profit immédiat des usagers bien sûr, mais aussi, sans doute, à celui de l'État.

Pour des forêts relativement denses, en bon état, les recettes peuvent peut-être être du même ordre. Mais les revenus que l'on peut tirer du parcours augmentent avec la dégradation de la forêt jusqu'à représenter plus de dix fois celles du bois, jusqu'à être enfin les seuls possibles.

Il est fréquent de constater que la charge du cheptel pesant sur un terroir, comprenant une forêt, paraît trop forte. II y a apparemment surpâturage. Malgré cela, l'utilisation des ressources fourragères d'une part, la gestion des troupeaux d'autre part, peuvent se caractériser par un important gaspillage.

Au cours de la saison des pluies, les troupeaux n'utilisent qu'une partie des ressources fourragères, alors abondantes et de qualité, disponibles en forêt ou sur certaines jachères. Puis avec la saison sèche, les aires du parcours sont peu à peu limitées par le tarissement des points d'eau. Le fourrage, non utilisé en forêt sèche, achève de devenir cellulosique, perdant une grande partie de sa valeur fourragère, devenant de plus en plus difficile à utiliser. On peut ainsi constater dans beaucoup de forêts, au milieu de la saison sèche, l'existence d'un volume important de fourrage, devenu pratiquement inutilisable, aliment pour les feux. C'est presque toujours la même chose pour certains résidus agricoles. La rotation du parcours, qui permettrait la mise en réserve d'une bonne partie de la production, pourrait entraîner un gros progrès.

En matière de gestion du cheptel, on constate une situation comparable. Beaucoup de troupeaux ne sont que des " tirelires " dont le rôle est d'attester une richesse et de payer certaines dépenses exceptionnelles. Mais leurs propriétaires ne recherchent pas la production optimum de viande, ou mieux la commercialisation optimum de celle produite.

Une bête, après avoir gagné du poids pendant l'hivernage, le perd souvent ainsi sans profit, pour survivre au cours de la saison sèche qui suit.

Dans pratiquement tout système pastoral englobant une forêt, pour ce qui nous concerne, il est certain qu'il existe de très importantes possibilités d'économie à organiser puis à mettre en œuvre. Le plus difficile reste le changement de mentalité des propriétaires des troupeaux et la formation des bergers. 
Ce n'est qu'après ces progrès que les autres techniques devraient être mises en œuvre: cultures fourragères intensives, améliorations pastorales extensives, ...

Le volant devenu disponible, grâce à une meilleure utilisation des ressources existantes, grâce à une meilleure gestion des troupeaux, puis grâce aux cultures fourragères intensives et aux améliorations pastorales extensives, devrait rendre possibles les mises en défens nécessaires aussi bien pour l'amélioration de la forêt que pour celle des parcours.

L'aménagement pastoral, qui inquiète beaucoup de forestiers, pourrait ainsi devenir un bon moyen pour intéresser et associer les populations, en leur faisant encaisser des recettes accrues, bénéfices d'une gestion raisonnée des parcours et des troupeaux.

$$
*^{*} *
$$

II est certain que l'aménagement des forêts soudano-sahéliennes est une nécessité pour gérer, c'est dire pour à la fois protéger, améliorer, produire. Existe-t-il d'autres moyens de sauver ces forêts que de les aménager et d'appliquer les aménagements ?

Et il est beaucoup plus facile, beaucoup moins coûteux de les sauver que de créer à grands frais sur un sol nu, érodé, des forêts nouvelles, d'autant plus que les populations voisines peuvent être associées à la mise en valeur, dans le cadre de contrats. Les usagers vont retirer, pour eux, les bénéfices de cette participation, en échange de prestations, qui contribuent encore plus à les associer à la forêt et au souci de sa pérennité.

Mais il n'y a pas d'aménagement-type susceptible d'être appliqué indifféremment à toutes les forêts. L'aménagement doit être "bâti " autour de la forêt, après que l'on ait bien compris le fonctionnement du système complexe qu'elle constitue. Cela suppose l'analyse de tous les facteurs intervenant pour expliquer la situation actuelle: le milieu, les populations et leurs troupeaux, sédentaires et nomades, et enfin la forêt elle-même, avec ses composantes forestières et pastorales. À partir de la situation actuelle, il faut essayer de prévoir la situation future.

Parmi les aménagements qui seront ainsi bâtis, certains ne pourront pas organiser d'exploitation, mais seulement des travaux d'enrichissement ou de reconstitution suivant la gravité de la dégradation. C'est pourtant dans la réalisation de ces travaux qu'il faudra le plus souvent rechercher les bénéfices que devront tirer les usagers de l'aménagement. II apparaît exceptionnel qu'il n'y en ait pas.

Et dans tous les aménagements, il faudra accorder une grande place à la régénération naturelle et aux dispositions qui la favorisent, quand elle est possible, sinon à l'enrichissement adapté ou à une reconstitution qui s'appuirait sur tous les lambeaux de l'ancien peuplement encore en place, soigneusement maintenus.

Ainsi conçus, une motivation essentielle des aménagements forestiers est de créer un certain état d'équilibre entre la forêt et les populations voisines, destiné à assurer la pérennité du système.

On comprend qu'il soit assez facile d'aménager une forêt quand la densité de la population tout autour est réduite $\left(15 / 20\right.$ habitants au $\left.\mathrm{km}^{2}\right)$ et qu'en conséquence les pressions exercées sont modérées.

Au fur et à mesure que la densité de la population augmente, que les besoins de toute nature croissent de même et que la forêt est davantage mise à contribution, rédiger un aménagement et surtout l'appliquer deviennent plus difficiles.

Très rapidement, il n'est plus possible d'aménager la forêt seule. II devient nécessaire de l'inclure dans l'aménagement de l'ensemble des ressources qui assurent la subsistance des 


\section{G. SOULĖRES}

populations. Et quelquefois, la permanence des peuplements boisés ne peut pas seulement être assurée par les interventions que l'on y réalise mais plutôt par celles que l'on peut conduire sur l'ensemble du terroir des usagers, dont certaines ont pour but de soulager la forêt.

Mais il est sûr que plus la densité de la population augmente, plus il devient difficile, complexe, d'aménager une forêt et plus l'aménagement doit devenir intégré, suivant un mot à la mode. Une bonne démarche peut ainsi être d'entreprendre très rapidement l'aménagement des forêts en bon état, encore entourées d'une population à faible densité, pour en faire des systèmes solides, capables, quand la population aura augmenté, de bien supporter l'évolution. C'est beaucoup plus facile que d'aménager sous la pression de la situation, pour éviter la disparition d'un massif, alors qu'il est déjà bien tard, ce que l'on est pourtant souvent obligé de faire.

G. SOULÈrES

Ingènieur des Eaux et Forêts

32, allée du Lac Superieur 78110 LE VÉSINET 